GTND

ISSN 0394-9362

\section{Nefrologo: agisci o perisci}

\section{Caro Direttore,}

complimenti per aver voluto, e con chiarezza, sollevare il problema dell'accesso vascolare in nefrologia.

Concordiamo pienamente sui numerosi punti emersi, ma su uno in particolare vorremmo focalizzare la tua attenzione e quella dei colleghi interessati all'argomento cioè l'idea di dar luogo a un team FAV (radiologo, nefrologo, chirurgo vascolare). Pur se bella e positiva, l'esperienza ci insegna che non sempre è realizzabile per i motivi che sinteticamente cercheremo di esporre.

1) I chirurghi vascolari sostanzialmente si sentono "sminuiti" nel confezionare una FAV. Cosa diversa potrebbe essere il coinvolgimento per step più complessi come impianti protesici, by-pass omero-radiale o nei casi di stenosi significativa e/o trombosi della vena succlavia ecc., dove la competenza, le attrezzature e gli eventuali risvolti legali suggeriscono di fare riferimento allo specialista.

2) Perché la PTA della vena cefalica la devono eseguire SOLO i radiologi interventisti e non il NEFROLOGO vista la possibilità di eseguirla sotto la guida ecografica? Un esempio ulteriore: perché la PTA specie del distretto arterioso periferico la può eseguire anche l'emodinamista e non solo il radiologo? Non potrebbe il nefrologo occuparsi anche del distretto cavale superiore? Non capisco come il vascolare, l'emodinamista ecc., possono occuparsene mentre il nefrologo no. La risposta è semplice: il NEFROLOGO negli ultimi anni ha giocato al ribasso e non ha invece rilanciato come hanno fatto le altre specialità, con i risultati evidenti che noi tutti conosciamo... il requiem della nefrologia (si ricordi il decreto Balduzzi-Lorenzin che prevede una UOC di Nefrologia ogni 0,6-1,2 milioni di abitanti).

Motivi di spazio non permettono ragionamenti articolati ma la domanda che dobbiamo porci è quale deve essere il ruolo del nefrologo in un ospedale; se da un lato le metodiche sostitutive ci hanno permesso di sopravvivere fino ad oggi, dall'altro la deospedalizzazione della cronicità a breve ci spoglierà anche di questo ramo della nostra disciplina. Per cui, se i trattamenti cronici andranno fuori dall'ospedale, i trattamenti continui ai rianimatori, gli accessi vascolari ai radiologi e chirurghi vascolari, l'imaging e la biopsia al radiologo e all'anatomopatologo e così via, a noi cosa rimane? II ruolo del coordinatore..., bene... per il ruolo di coordinatore non è necessaria una UOC. Accanto alla biopsia renale, al metabolismo calcio-fosforo, all'anemia ecc., il nefrologo del presente/ futuro deve avere una importante e dilagante componente interventistica. Potremmo essere, per la storia della disciplina e per la cultura che abbiamo, gli unici internisti completi e interventisti... figura di cui in ospedale si ha veramente bisogno. Il team AV per alti livelli di difficoltà è auspicabile, ma è anche vero che la creazione, ad esempio, dell'ambulatorio dell'accesso vascolare nelle nostre UOC potrebbe drasticamente ridurre la necessità di disturbare gli altri colleghi con gli immaginabili benefici per i nostri pazienti.

Concludiamo dicendo che a NOI e solo a NOI è affidato il futuro della Nefrologia e quindi continuiamo a fare biopsie, FAV di I-II livello, posizionamento dei CVC, toracentesi, nefrostomie, posizionamento di cateteri in peritoneo ecc.: facciamoci contaminare dalle abilità dei colleghi... basta solo volerlo!

\section{Disclosures}

Financial support: No financial support was received for this submission. Conflict of interest: The authors haven't conflict of interest.

\section{Antonio Granata ${ }^{1}$, Pasquale Fatuzzo ${ }^{2}$}

${ }^{1}$ UOC Nefrologia e Dialisi, PO "San Giovanni di Dio", Agrigento

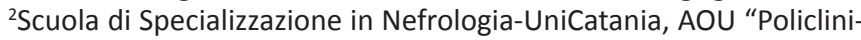
co-Vittorio Emanuele", Catania

Indirizzo per la corrispondenza:

Dr. Antonio Granata

UOC Nefrologia e Dialisi, PO "San Giovanni di Dio"

C/da Consolida

92100 Agrigento

antoniogranata4@tim.it

Published online: May 16, 2017 